\title{
Características físico-químicas e sensoriais de batata frita da cultivar BRS Ana branqueada e revestida com metilcelulose
}

\author{
Physicochemical and sensory characteristics of potato chips made from blanched \\ potatoes of the cultivar BRS Ana and coated with methylcellulose
}

\section{Autores | Authors}

*Márcia de Mello LUVIELMO

Universidade Federal do Rio Grande

(FURG)

Escola de Química e Alimentos

Campus Universitário Carreiros,

Av. Itália, km 8

CEP: 96203-900

Rio Grande/RS - Brasil

e-mail:mmluvielmo@gmail.com

\section{Caroline Dellinghausen BORGES} Marina Vighi SCHIAVON

Daniela Silva DE ARMAS

Flávia Fernandes PAIVA

Universidade Federal de Pelotas (UFPel) Centro de Ciências Químicas, 'Farmaceuticas e de Alimentos Pelotas/RS - Brasi e-mail: caroldellin@bol.com.br marinavighi@gmail.com armasdd@hotmail.com fafernadespaiva@yahoo.com.br

\section{Ana Cristina Richter KROLOW Núbia Marilin Lettinin FERRI \\ EMBRAPA-Clima Temperado Pelotas/RS - Brasi e-mail: ana.krolow@embrapa.br nubia.ferri@embrapa.br}

*Autor Correspondente / Corresponding Author

Recebido: Jun. 24, 2015

Aprovado: Nov. 04, 2015

\section{Resumo}

O estudo propôs avaliar o efeito do branqueamento combinado ao uso de sais e de revestimentos à base de metilcelulose $(\mathrm{MC})$ na absorção de gordura e nas características físico-químicas e sensoriais de batatas da cultivar BRS Ana, submetidas à fritura na forma de batata chips. O experimento foi realizado com a cultivar BRS Ana e a cultivar Asterix, sendo a batata Asterix adequada para produtos fritos. Para o estudo foram definidos controles para as batatas das cultivares BRS Ana (TO') e Asterix (TO"), batatas que não foram submetidas ao branqueamento nem a qualquer tipo de revestimento. Foram testados três tratamentos para cada cultivar; para a cultivar BRS Ana: T1: com branqueamento e sem revestimento; T2: com branqueamento e com revestimento de $1 \%$ de MC; T3: com branqueamento e com revestimento de $2 \%$ de MC; para a cultivar Asterix: T4: com branqueamento e sem revestimento; T5: com branqueamento e com revestimento de $1 \%$ de MC; T6 com branqueamento e com revestimento de $2 \%$ de MC. Para os tratamentos que receberam o branqueamento (T1, T2, T3, T4, T5 e T6), foi realizada uma imersão das fatias de batata em água a $85^{\circ} \mathrm{C}$ por 3 minutos, com adição de $0,5 \%$ de $\mathrm{CaCl}_{2}$ e 2,5\% de $\mathrm{NaCl}$, seguida de resfriamento e centrifugação. As batatas dos tratamentos T2, T3, T5 e T6 que receberam revestimento de MC foram imersas em solução de MC 1 ou $2 \%(p / v)$, por 1 min e, em seguida, drenadas. As batatas foram submetidas à fritura em gordura vegetal a $180^{\circ} \mathrm{C}$. A maior redução de absorção de gordura (13\%) durante a fritura foi para batata da cultivar BRS Ana submetida ao branqueamento e revestimento com $1 \% \mathrm{MC}$, obtendo-se no produto final $29,37 \%$ de gordura. Além disso, o revestimento com $1 \%$ MC não modificou significativamente a cor e a textura das batatas chips; nos resultados sensoriais esse tratamento não influenciou na preferência para os atributos testados (aparência, cor, sabor e textura) e o índice de aceitação sensorial desse tratamento foi de $85,9 \%$. A batata da cultivar BRS Ana submetida ao branqueamento adicionado de $\mathrm{NaCl}$ e $\mathrm{CaCl}_{2}$ e revestimento com $1 \%$ de $\mathrm{MC}$ apresenta potencial de utilização na forma de batata chips.

Palavras-chave: Batata chips; Absorção de gordura; Goma; Aceitação; Batata cultivar BRS Ana.

\section{Summary}

The present study aimed to evaluate the effect of blanching combined with the use of salts and methylcellulose coatings (MC) on fat absorption and the physicochemical and sensory characteristics of potatoes of the cultivar BRS Ana, subjected to frying in the form of chips. The experiment was carried out with the cultivars BRS Ana and Asterix, Asterix potatoes being adequate for fried products. The control samples for the cultivars BRS Ana (TO') and Asterix (TO") were not subjected to either blanching or any type of coating. Three treatments were tested for each cultivar, as follows: for the cultivar BRS Ana: T1: with blanching, without coating; T2: with blanching and coating using 1\% MC; T3: with blanching and coating using $2 \% \mathrm{MC}$; for the cultivar Asterix: T4: with blanching, without coating; $\mathrm{T} 5$ : with blanching and coating using $1 \% \mathrm{MC}$; $\mathrm{T} 6$ with blanching and coating using $2 \% \mathrm{MC}$. For the treatments subjected to blanching ( $T 1, T 2, T 3, T 4, T 5$ and $T 6)$, the sliced potatoes were immersed in water at $85^{\circ} \mathrm{C}$ for 3 minutes with the addition of $0.5 \% \mathrm{CaCl}_{2}$ and $2.5 \% \mathrm{NaCl}$, followed by cooling and centrifugation. The potatoes 
used in treatments T2, T3, T5 and T6, which were coated with MC, were immersed in 1 or $2 \%(\mathrm{w} / \mathrm{v}) \mathrm{MC}$ solution for 1 minute and then drained. All the potato chips were subjected to frying in vegetable oil at $180^{\circ} \mathrm{C}$. The cultivar BRS Ana subjected to blanching and coating with $1 \% \mathrm{MC}$ showed the lowest fat absorption (13\%) during frying, with $29.37 \%$ fat in the final product. Furthermore, coating with $1 \%$ MC did not significantly affect the attributes of colour and texture of the potato chips or the consumer preference for the attributes studied (appearance, colour, flavour, and texture), with a sensory acceptance score of $85.9 \%$. The cultivar BRS Ana potatoes subjected to blanching with the addition of $\mathrm{NaCl}$ and $\mathrm{CaCl}_{2}$ and coated with $1 \% \mathrm{MC}$ showed good potential for use in the form of potato chips.

Key words: Potato chips; Fat absorption; Gum; Acceptance; Cultivar BRS Ana potatoes.

\section{Introdução}

A industrialização da batata no Brasil cresceu a partir da década de 90 , primeiramente para a fabricação de batata frita na forma de rodelas (chips), posteriormente na forma de batata palha. Em 2006, foi estabelecida a industrialização, em escala, da batata na forma de palitos pré-fritos congelados, visando diminuir a importação, que em 2010 foi de cerca de 200 mil toneladas. Em 2011, a batata industrializada atingiu $30 \%$ do produto consumido (PEREIRA, 2011). Em 2014, a produção de batata no Brasil foi de 3.569.750 toneladas (IBGE, 2014).

O crescimento do consumo de produtos industrializados de batata no Brasil tem sido limitado quase que exclusivamente pela não disponibilidade de matérias-primas adequadas à industrialização (FREITAS et al., 2006). A qualidade do produto processado é dependente de altos teores de matéria seca, que reduz a absorção de óleo durante a fritura e confere crocância, e de baixos teores de açúcares redutores, que mantêm a coloração clara das fritas (FREITAS et al., 2006).

No processamento de batata frita, o processo de fritura consiste na imersão do produto em óleo sobre altas temperaturas, aproximadamente 180 a $200{ }^{\circ} \mathrm{C}$, por um curto período de tempo (JORGE; LUNARDI, 2005; DEL RÉ; JORGE, 2006). À medida que começa a ebulição da água da superfície do produto, a convecção é intensificada pelo vapor de água turbulento devido à evaporação, assim irá ocorrer a secagem da superfície. A evaporação também ocasiona o encolhimento e desenvolvimento de porosidade e rugosidade (MELLENA, 2003).

Quando a temperatura da superfície da batata eleva-se acima da temperatura de ebulição da água, ocorrem várias alterações físico-químicas, como a retrogradação do amido e a reação de Maillard, o que ocasiona efeitos benéficos em relação às características sensoriais, como o desenvolvimento da cor e a formação da crosta, originando um produto com cobertura apreciada (MELLENA, 2003).
A absorção de óleo em batatas fritas oscila de 0,2 a 0,7 g de óleo/g sólidos secos (PEDRESCHI; MOYANO, 2005; PEDRESCHI et al., 2007, 2008). Nesse sentido, a redução do conteúdo de lipídios nos alimentos fritos é requerida, principalmente, devido a sua relação com doenças coronarianas e a obesidade (SUÁREZ et al., 2008).

O grau de absorção de óleo é significativamente afetado por fatores como os pré-tratamentos aplicados ao alimento, as características físico-químicas do alimento, as condições do processo (temperatura, tempo), a origem e composição química do óleo, entre outros (RIMAC-BRNCIC et al., 2004; TRONCOSO et al., 2009).

Além destes, a cultivar da batata é outro fator que influencia na absorção de óleo durante a fritura. Os pesquisadores do Programa de Melhoramento de Batata da Embrapa (Clima Temperado, Pelotas/RS, Transferência de Tecnologia/EN-Canoinhas/SC e Hortaliças, Brasília/DF) desenvolveram uma nova cultivar de batata, denominada BRS Ana, que absorve menor quantidade de gordura, sendo adequada para fritura à francesa, com potencial de processamento na forma de palitos pré-fritos congelados e de flocos (PEREIRA et al., 2010).

As batatas das cultivares Asterix e Agata são as mais cultivadas no Brasil (PEREIRA et al., 2008). No estudo realizado por Fernandes et al. (2010), a cultivar Agata apresentou características adequadas para o mercado fresco, sendo indicada para a preparação de massas e pratos assados. A cultivar Asterix apresenta teor satisfatório de matéria seca para frituras doméstica e industrial, na forma de palitos. Inclusive, essa cultivar tem sido a fonte básica de matéria-prima para a crescente industrialização de batata na forma de palitos pré-fritos congelados no país (PEREIRA, 2011).

Como pré-tratamento para produtos que serão submetidos à fritura, tem sido preconizada a aplicação de branqueamento, uso de sais e revestimentos com hidrocoloides (ZENG et al., 2010; KIM et al., 2011; HUA et al., 2015). 
De acordo com Vendruscolo e Zorzella (2002), a etapa de branqueamento melhora a qualidade da batata quanto à textura, promovendo a gelatinização do amido, o que resulta em menor absorção de gordura. Também melhora a cor, uma vez que remove parte de substâncias que provocam o escurecimento. Outras alternativas para reduzir a absorção de gordura são a imersão da batata em uma solução de $\mathrm{NaCl}$ (BUNGER et al., 2003; PEDRESCHI et al., 2007) e o branqueamento associado à adição de $\mathrm{NaCl}_{2}$ (RIMAC-BRNCIC et al., 2004).

Os hidrocoloides têm sido utilizados como revestimentos devido às propriedades de barreira a gases e vapor de água, propriedades mecânicas e barreira à gordura, o que reduz a absorção de óleo durante a fritura (GARCÍA et al., 2002). Dentre os principais hidrocoloides utilizados com este propósito destacam-se os derivados da celulose como a metilcelulose (MC) (ALBERT; MITTAL, 2002; GARCÍA et al., 2002; SUÁREZ et al., 2008; DIAS, 2011), carboximetilcelulose (RIMAC-BRNCIC et al., 2004), hidroximetilcelulose (GARCÍA et al., 2002), além do alginato (ZENG et al., 2010), pectina (ALBERT; MITTAL, 2002; ZENG et al., 2010; HUA et al., 2015), carragena (ALBERT; MITTAL, 2002), gelana (ALBERT; MITTAL, 2002; KIM et al., 2011), guar (KIM et al., 2011), locusta (ALBERT; MITTAL, 2002) e xantana (ZENG et al., 2010).

O efeito de hidrofilicidade, propriedades espessantes e gelificação térmica são características requeridas para esses hidrocoloides (GARCÍA et al., 2002; $\mathrm{KIM}$ et al., 2011). A MC apresenta um comportamento incomum, pois possui a capacidade de gelificar-se reversivelmente sob o efeito do calor $\left(50-70^{\circ} \mathrm{C}\right)$ e o gel se rompe após o resfriamento, além de ser solúvel em água fria, insolúvel em gordura, sem qualquer valor calórico, cor e sabor (PENA, 1999).

O presente estudo propôs avaliar o efeito do branqueamento combinado ao uso de sais e de revestimentos à base de metilcelulose na absorção de gordura, características físico-químicas e sensoriais de batatas da cultivar BRS Ana submetidas à fritura na forma de batata chips.

\section{Material e métodos}

\subsection{Material}

As batatas utilizadas foram das cultivares BRS Ana e Asterix doadas pela Embrapa Clima Temperado, Pelotas/RS, Brasil. Estas foram transportadas para o Laboratório de Processamento de Alimentos do Centro de Ciências Químicas, Farmacêuticas e de Alimentos da Universidade Federal de Pelotas, onde foram submetidas ao processamento. Foi utilizada a metilcelulose M0262 (Sigma Aldrich) que, a $2 \%$ em água $\left(20^{\circ} \mathrm{C}\right)$, apresenta 400 cP de viscosidade.

\subsection{Tratamentos e processamento das batatas fritas}

\subsubsection{Tratamentos}

Foram realizados oito tratamentos:

BRS Ana (TO'): Controle - batata da cultivar BRS Ana sem branqueamento e sem revestimento;

BRANQ (T1): batata da cultivar BRS Ana com branqueamento e sem revestimento;

1\%MC (T2): batata da cultivar BRS Ana com branqueamento e com revestimento de $1 \%$ de MC;

2\%MC (T3): batata da cultivar BRS Ana com branqueamento e com revestimento de $2 \%$ de $M C$;

ASTERIX (TO"): Controle - batata da cultivar Asterix sem branqueamento e sem revestimento;

BRANQ (T4): batata da cultivar Asterix com branqueamento e sem revestimento;

1\%MC (T5): batata da cultivar Asterix com branqueamento e com revestimento de $1 \%$ de MC;

2\%MC (T6): batata da cultivar Asterix com branqueamento e com revestimento de $2 \%$ de MC.

O delineamento experimental utilizado foi inteiramente ao acaso em esquema fatorial $2 \times 8 \times 1$, sendo duas variedades de batatas (BRS Ana e Asterix), 8 tratamentos (T0', T1, T2, T3, T0", T4, T5 e T6) e 1 período de avaliação.

\subsubsection{Processamento}

Foram utilizadas 42 batatas de cada cultivar, estas foram selecionadas quanto à ausência de defeitos fisiológicos, tamanho e cor; após, foram lavadas, sanitizadas com solução de hipoclorito de sódio na concentração de 150 ppm por 15 min, descascadas manualmente e cortadas com o auxílio de um cortador rotativo regulado para formato chips, de aproximadamente 2 mm. Em seguida, para a remoção do excesso de amido, os pedaços foram imersos em água por $1 \mathrm{~min}$.

\subsubsection{Branqueamento}

Para os tratamentos que receberam o branqueamento ( $T 1, T 2, T 3, T 4, T 5$ e T6), este foi realizado pela imersão dos pedaços de batata em água a $85{ }^{\circ} \mathrm{C}$ por $3 \mathrm{~min}$, com adição de 0,5\% de $\mathrm{CaCl}_{2}$ e 2,5\% de $\mathrm{NaCl}$, após, as batatas foram resfriadas e centrifugadas manualmente por $2 \mathrm{~min}$. 


\subsubsection{Revestimentos com MC}

Para os tratamentos que receberam o revestimento de MC (T2, T3, T5 e T6), seguindo o processamento, as batatas foram imersas, à temperatura ambiente por $1 \mathrm{~min}$, em solução aquosa de MC 1\% (T2 e T5) ou 2\% (T3 e T6) $(\mathrm{p} / \mathrm{v})$, adicionadas de $0,75 \%(\mathrm{v} / \mathrm{v})$ de glicerol. Após esse período as fatias foram distribuídas em telas de náilon para que o excesso da solução fosse drenado.

\subsubsection{Fritura}

As batatas de cada tratamento foram submetidas separadamente à fritura em fritadeira elétrica semi-industrial. A gordura vegetal para fritura foi aquecida a $180{ }^{\circ} \mathrm{C}$. O ponto final foi verificado no momento em que cessou o desprendimento das últimas bolhas da superfície da batata.

\subsection{Avaliações}

\subsubsection{Teor de gordura}

O teor de gordura foi determinado em triplicata, em aparelho extrator de Soxhlet, conforme metodologia Cunniff (1995) método 920.39,C, utilizando para a extração éter etílico por um tempo de 6h. Para o cálculo do percentual de variação no teor gordura (\%VG) utilizou-se a Equação 1 (ALBERT; MITTAL, 2002).

$\% V G=\frac{G_{\text {controle }}-G_{\text {amostra }}}{G_{\text {controle }}} \times 100$

\subsubsection{Teor de glicose}

O teor de glicose foi determinado, em triplicata, segundo metodologia de Somogyi, adaptada por Nelson (1944).

Para tal determinação, foi pesado $25 \mathrm{~g}$ de amostra, adicionado de $50 \mathrm{~mL}$ de água, após, submeteu-se à homogeneização com o auxílio de um ultra-turrax. Adicionou-se 2,5 $\mathrm{mL}$ de $\mathrm{NaOH} 1 \mathrm{M}$, seguiu-se a neutralização com ácido acético glacial concentrado e todo o volume foi transferido para balão volumétrico e completado o volume (250 mL). Filtrou-se a vácuo (extrato). Foi realizada a desproteinização; para isso, em $5 \mathrm{~mL}$ do extrato (filtrado) foi adicionado $1,2 \mathrm{~mL}$ de $\mathrm{Ba}(\mathrm{OH})_{2}$, $1,2 \mathrm{~mL}$ de $\mathrm{ZnSO}_{4}$ e $9 \mathrm{~mL}$ de $\mathrm{H}_{2} \mathrm{O}$, agitou-se e permaneceu em repouso por 10 minutos, seguido de filtração, deste $1,0 \mathrm{~mL}$, foi adicionado e $1,0 \mathrm{~mL}$ de $\mathrm{H}_{2} \mathrm{O}, 1,0 \mathrm{~mL}$ do reativo cúprico e posto em banho-maria fervente por $20 \mathrm{~min}$, seguido de resfriamento em água gelada. Acrescentou-se $1 \mathrm{~mL}$ de reativo arsenomolibídico e completou-se o volume final dos tubos para $10 \mathrm{~mL}$ usando $6 \mathrm{~mL}$ de $\mathrm{H}_{2} \mathrm{O}$. Foi realizada leitura em espectrofotômetro a $510 \mathrm{~nm}$. A curva com glicose p.a. foi realizada utilizando-se soluções de glicose com $100 \mathrm{mg} / \mathrm{mL}, 0,2 \mathrm{~mL} ; 0,4 \mathrm{~mL}$; 0,6mL; 0,8 mL; 1,0 mL; 1,2 mL.

\subsubsection{Determinação de umidade}

O teor de umidade foi determinado em triplicata, por gravimetria em estufa a vácuo a $70{ }^{\circ} \mathrm{C}$, segundo Cunniff (1995), método 984.25. Para o cálculo do percentual de umidade retida (\%UR) utilizou-se a Equação 2, adaptado de Albert e Mittal (2002).

$\% U R=\frac{U R_{\text {controle }}-U R_{\text {amostra }}}{U R_{\text {controle }}} \times 100$

\subsubsection{Propriedade mecânica}

A força de quebra das amostras de batata frita foi analisada em texturômetro TA.XT plus, utilizando o software Exponent Stable Micro System. As batatas foram selecionadas de forma aleatória e colocadas horizontalmente na plataforma, utilizando-se lâmina de aço retangular de ponta arredondada para cortá-las ao meio. As condições do teste foram: velocidade de pré-teste $2 \mathrm{~mm} \cdot \mathrm{s}^{-1}$, pós-teste $10 \mathrm{~mm} \cdot \mathrm{s}^{-1}$, de teste $1 \mathrm{~mm} \cdot \mathrm{s}^{-1}$. Os resultados representam a média de 10 determinações. A força de quebra correspondeu à altura do primeiro pico significativo, no primeiro ciclo de compressão.

\subsubsection{Cor}

As análises de cor foram realizadas utilizando-se um colorímetro (Minolta, modelo Chroma Meter CR400). Foram verificados os parâmetros de luminosidade $L^{*}$ [0 (preto) a 100 (branco)], a* [cromaticidade do verde $(-60)$ a vermelho $(+60)$ ] e b* [cromaticidade do azul $(-60)$ para amarelo $(+60)]$. Foram analisadas 6 amostras de cada tratamento, sendo realizadas 3 leituras por amostra.

\subsubsection{Análise sensorial}

Os procedimentos de avaliação sensorial ocorreram em cabines individuais no Laboratório de Análise Sensorial do Centro de Ciências Químicas, Farmacêuticas e de Alimentos da Universidade Federal de Pelotas.

\subsubsection{Teste de ordenação de preferência}

Para a realização da análise sensorial foram excluídos os tratamento controle (TO' e TO"). Os seis tratamentos (T1, T2, T3, T4, T5 e T6) foram submetidos ao teste de ordenação de preferência por 50 avaliadores familiarizados com o teste (DUTCOSKY, 2013). Foi solicitado que os avaliadores ordenassem os tratamentos do menos preferido (1 - menos preferido) para o mais preferido (6 - mais preferido). As amostras foram apresentadas simultaneamente, em ordem que segue delineamento de blocos completos casualizados. 
Foram servidas em recipientes de porcelana branca codificados com números de três dígitos aleatórios, na quantidade de três unidades/avaliador, à temperatura ambiente) (DUTCOSKY, 2013). Os atributos solicitados para a ordenação de preferência foram sabor, cor, aparência, textura e preferência global.

\subsubsection{Teste de aceitação}

O tratamento que obteve o menor percentual de absorção de gordura foi submetido ao teste de aceitação. Para o teste de aceitação uma escala hedônica de nove pontos foi apresentada a 78 avaliadores não treinados (DUTCOSKY, 2013). Os atributos solicitados para avaliação quanto à aceitação foram sabor, aroma, aparência, textura e a aceitação global do produto. Os resultados foram analisados pelo índice de aceitabilidade conforme metodologia de Dutcosky (2013).

O trabalho foi aprovado pelo Comitê de Ética e Pesquisa da Universidade Federal de Pelotas sob o número CAAE: 36441414.6.0000.5317.

\subsubsection{Avaliação estatística}

Os dados foram analisados usando o software (STATISTIX 10, 2014). Os resultados obtidos para as avaliações físico-químicas foram submetidos à análise de variância one-way (ANOVA), seguido pelo teste de Tukey para comparação das médias, com nível de significância de $p \leq 0,05$ e nível de confiança de 95\%.

Em relação aos resultados obtidos pelo teste sensorial de ordenação de preferência, os mesmos foram analisados estatisticamente através do método de Friedman e tabela de Newell e MacFarlane (DUTCOSKY, 2013), com nível de significância de $p \leq 0,05$ e nível de confiança de $95 \%$.

\section{Resultados e discussão}

As batatas in natura, das cultivares BRS Ana e Asterix, apresentaram teor de umidade de 78,75\% e $76,20 \%$, respectivamente. De acordo com os resultados (Tabela 1), pode-se observar que após o processo de fritura, a batata frita BRS Ana (TO') apresentou, significativamente $(p \leq 0,05)$, maior teor de umidade e menor quantidade de gordura que a cultivar Asterix (TO"), como esperado.

De acordo com Garayo e Moreira (2002), o conteúdo de gordura da batata chips submetida à fritura varia de 35,3 a 44,5\%. Zorzella et al. (2003) ao processarem, na forma de chips, treze diferentes genótipos de batata (entre eles 5 cultivares e 8 clones avançados), encontraram teores de gordura após o processo de fritura entre 32,99 e 41,20\%. No presente estudo, valores inferiores foram obtidos, o que pode estar relacionado à cultivar da batata, ao pré-tratamento e à concentração de MC aplicada.

A batata BRS Ana processada na forma de chips submetida à fritura apresentou menor teor de gordura em relação à batata Asterix, esta característica foi observada no tratamento controle (BRS Ana - T0' em relação à Asterix - T0"), assim como em todos os tratamentos em que houve o revestimento com MC (BRS Ana - T2, T3 em relação à Asterix - T5, T6). Comportamento semelhante foi obtido por Pereira et al. (2010), ao avaliarem as batatas BRS Ana em relação às batatas das cultivares Asterix e Agata.

Com a finalidade de reduzir ainda mais a absorção de gordura, o branqueamento com a adição de sais foi testado. Foi possível observar que o branqueamento combinado com a adição de sais proporcionou significativamente $(p \leq 0,05)$ menor absorção de gordura e maior perda de umidade em ambas as cultivares

Tabela 1. Teor de umidade e gordura de batatas chips fritas das cultivares BRS Ana e Asterix submetidas a diferentes tratamentos.

\begin{tabular}{|c|c|c|c|c|c|c|c|}
\hline \multirow{2}{*}{$\begin{array}{l}\text { Cultivar } \\
\text { Batata }\end{array}$} & \multirow{2}{*}{ Tratamento } & \multirow{2}{*}{$\begin{array}{l}\text { Umidade } \\
\qquad(\%)\end{array}$} & \multicolumn{2}{|c|}{$\begin{array}{c}\text { Variação no conteúdo de } \\
\text { umidade (\%) }\end{array}$} & \multirow{2}{*}{$\begin{array}{c}\text { Gordura } \\
(\%)\end{array}$} & \multicolumn{2}{|c|}{$\begin{array}{c}\text { Variação no teor de gordura } \\
(\%)\end{array}$} \\
\hline & & & $\begin{array}{c}\text { relação ao } \\
\text { T1 e T4 }\end{array}$ & $\begin{array}{l}\text { relação ao } \\
\text { TO' e T0" }\end{array}$ & & $\begin{array}{c}\text { relação ao } \\
\mathrm{T} 1 \text { e T4 }\end{array}$ & $\begin{array}{l}\text { relação a } \\
\text { TO' e T0" }\end{array}$ \\
\hline \multirow{4}{*}{ BRS ANA } & Controle(TO') & $8,52^{a}$ & & $x$ & $33,95^{b}$ & & $x$ \\
\hline & BRANQ.(T1) & $4,69^{d}$ & $x$ & & $30,62^{\text {de }}$ & $x$ & \\
\hline & 1\%MC (T2) & $4,26^{e}$ & $-9,16$ & $-50,00$ & $29,37^{\dagger}$ & $-4,08$ & $-13,49$ \\
\hline & 2\%MC (T3) & $4,19^{e}$ & $-10,66$ & $-50,82$ & $31,23^{d}$ & $+1,99$ & $-8,01$ \\
\hline \multirow{4}{*}{ ASTERIX } & Controle(TO") & $6,16^{b}$ & & $x$ & $38,75^{a}$ & & $x$ \\
\hline & BRANQ.(T4) & $5,66^{c}$ & $x$ & & $30,42^{\mathrm{e}}$ & $x$ & \\
\hline & 1\%MC (T5) & $4,22^{e}$ & $-25,44$ & $-31,49$ & $33,93^{b}$ & $+11,53$ & $-12,43$ \\
\hline & 2\%MC (T6) & $4,35^{e}$ & $-23,14$ & $-29,38$ & $32,20^{c}$ & $+5,85$ & $-16,90$ \\
\hline
\end{tabular}

Médias seguidas de mesma letra minúscula na coluna não diferem entre si, pelo Teste de Tukey $(p \leq 0,05)$. BRS Ana: Controle (To') = batata sem branqueamento e sem revestimento; BRANQ (T1) = batata com branqueamento e sem revestimento; $1 \%$ MC (T2) = batata com branqueamento e com revestimento de $1 \%$ de MC; $2 \%$ MC (T3) = batata com branqueamento e com revestimento de $2 \%$ de MC; ASTERIX: Controle (TO") = batata sem branqueamento e sem revestimento; BRANQ (T4) = batata com branqueamento e sem revestimento; $1 \%$ MC (T5) = batata com branqueamento e com revestimento de $1 \%$ de $\mathrm{MC} ; 2 \% \mathrm{MC}(\mathrm{T} 6)$ = batata com branqueamento e com revestimento de $2 \%$ de $\mathrm{MC}$. 
de batata (T1 e T4); em relação à batata que não foi submetida ao branqueamento (TO' e TO"), entretanto, não houve diferença significativa no teor de gordura entre as cultivares (T1 e T4) após o branqueamento (Tabela 1).

Redução de 22,2\% na absorção de gordura na batata palito foi obtida no estudo realizado por Bunger et al. (2003) com a imersão das batatas em solução a $3 \%$ de $\mathrm{NaCl}$ a $25{ }^{\circ} \mathrm{C}$ após o branqueamento. Já Rimac-Brncic et al. (2004) obtiveram redução máxima de gordura de 27-28\% ao aplicar o branqueamento juntamente ao $\mathrm{CaCl}_{2}$ em batatas palito, obtendo teor final de gordura de 30,76\%, quando utilizado óleo vegetal. Segundo os autores, a adição de $\mathrm{CaCl}_{2}$ na água de branqueamento reduz a absorção de gordura, pois este sal estabiliza os tecidos durante o processo de fritura, devido à reação entre a pectina da batata e o cálcio, promovendo a manutenção da textura (firmeza e rigidez). Os resultados de redução de absorção de gordura foram superiores aos obtidos neste estudo, entretanto, o teor final de gordura para a batata BRS Ana foi inferior $(29,37 \%)$.

Com relação à aplicação da MC, comportamento diferenciado foi observado nas diferentes cultivares de batatas. A batata BRS Ana apresentou, significativamente, menor teor de gordura $(29,37 \%)$ quando utilizado $1 \%$ de MC (T2), correspondendo a uma redução de $4,08 \%$ em relação ao tratamento em que a batata foi submetida ao branqueamento e sem revestimento (T1). Quando comparado ao tratamento em que a batata não foi submetida ao branqueamento (TO'), a redução foi superior, alcançando 13,49\%.

No tratamento em que a batata desta cultivar foi revestida com 2\% de MC (T3), pôde-se observar aumento no teor de gordura $(31,23 \%)$, correspondendo a um acréscimo de 1,99\% em relação ao T1 e redução de 8,01\% em relação ao T0'.
Já nas batatas da cultivar Asterix pôde-se observar aumento significativo ( $p \leq 0,05)$ no teor de gordura nas amostras revestidas com 1 e $2 \%$ de $\mathrm{MC}$, alcançando teor de gordura de 33,93 e $32,20 \%$, o que corresponde a um aumento de 11,53 e 5,85\%, respectivamente, em relação ao tratamento $\mathrm{T} 4$, em que a batata foi submetida ao branqueamento e sem revestimento. Mas em relação ao tratamento T0", em que a batata não foi submetida ao branqueamento, houve redução na absorção de gordura de 12,43\% (T5) e 16,90\% (T6).

Os revestimentos com $\mathrm{MC}$ ( 1 e $2 \%$ ), independente da cultivar de batata, não foram eficientes na manutenção do teor de umidade, visto que ocorreu redução significativa nos valores, após os tratamentos.

Os valores obtidos no teor de gordura para as batatas BRS Ana submetidas ao branqueamento e adicionadas de $1 \%$ de MC estão condizentes com os valores relatados na literatura para a batata chips, entretanto, a porcentagem de redução foi inferior, possivelmente em função da cultivar da batata. No estudo realizado por Tran et al. (2007), houve redução de 30\% na absorção de gordura de batatas chips submetidas à combinação de processamento branqueamento+secagem+imersão em solução de açúcar, alcançando um teor final de gordura de $26,4 \%$.

Revestimentos à base de MC são efetivos na redução da absorção de gordura, por exibirem termo-geleificação. Tavera-Quiroz et al. (2012), aplicando metilcelulose (10 g. $\left.\mathrm{L}^{-1}\right)$ na mesma concentração utilizada neste estudo e adição de $7,5 \mathrm{~g} \cdot \mathrm{L}^{-1}$ de plastificante, obtiveram como resultado uma redução na absorção de óleo de 30\% e teor final de gordura de, aproximadamente, $25 \%$ na batata chips.

De acordo com os resultados de força de quebra apresentados na Tabela 2, as amostras de batata BRS Ana submetidas aos tratamentos T0', T1, T2 e T3 apresentaram valores médios superiores aos da cultivar

Tabela 2. Força de quebra, $L^{*}, a^{*}$ e $b^{*}$ de batatas chips fritas das cultivares BRS Ana e Asterix submetidas a diferentes tratamentos.

\begin{tabular}{|c|c|c|c|c|c|}
\hline Cultivar Batata & Tratamento & $\begin{array}{c}\text { Força de quebra } \\
\text { (N) }\end{array}$ & $L^{*}$ & $a^{*}$ & $\mathbf{b}^{*}$ \\
\hline \multirow{4}{*}{ BRS ANA } & Controle(TO') & $3,50^{\mathrm{ab}}$ & $61,48^{a}$ & $1,60^{a}$ & $29,83^{a}$ \\
\hline & BRANQ.(T1) & $5,13^{a}$ & $55,45^{b}$ & $-1,79^{c}$ & $24,55^{\text {bc }}$ \\
\hline & 1\%MC (T2) & $4,80^{\mathrm{ab}}$ & $56,48^{a b}$ & $-2,76^{c}$ & $22,61^{c}$ \\
\hline & 2\%MC (T3) & $5,20^{a}$ & $56,11^{\mathrm{ab}}$ & $-0,12^{a b c}$ & $24,71^{\text {bc }}$ \\
\hline \multirow{4}{*}{ ASTERIX } & Controle(TO") & $3,01^{b}$ & $54,27^{b}$ & $1,25^{a b}$ & $25,34^{\text {bc }}$ \\
\hline & BRANQ.(T4) & $3,89^{a b}$ & $53,66^{b}$ & $-2,08^{c}$ & $24,58^{\text {bc }}$ \\
\hline & 1\%MC (T5) & $3,36^{a b}$ & $56,30^{\mathrm{ab}}$ & $-2,50^{c}$ & $24,24^{\text {bc }}$ \\
\hline & $2 \% \mathrm{MC}(\mathrm{T} 6)$ & $4,14^{\mathrm{ab}}$ & $56,53^{\mathrm{ab}}$ & $-1,46^{b c}$ & $26,60^{\mathrm{ab}}$ \\
\hline
\end{tabular}

Médias seguidas de mesma letra minúscula na coluna não diferem entre si, pelo Teste de Tukey $(p \leq 0,05)$. BRS Ana (TO'): Controle - batata sem branqueamento e sem revestimento; BRANQ (T1): batata com branqueamento e sem revestimento; 1\%MC (T2): batata com branqueamento e com revestimento de $1 \%$ de MC; $2 \%$ MC (T3): batata com branqueamento e com revestimento de $2 \%$ de MC; ASTERIX (TO"): Controle - batata sem branqueamento e sem revestimento; BRANQ (T4): batata com branqueamento e sem revestimento; $1 \%$ MC (T5): batata com branqueamento e com revestimento de $1 \%$ de $\mathrm{MC} ; 2 \% \mathrm{MC}$ (T6): batata com branqueamento e com revestimento de $2 \%$ de $\mathrm{MC}$. 
Asterix (T0", T4, T5, T6), entretanto, sem diferença significativa. O comportamento observado foi semelhante para as duas cultivares. O branqueamento associado aos sais não proporcionou um aumento significativo na força necessária para quebrar a batata frita.

No estudo realizado por Pedreschi e Moyano (2005), também não foi observada influência do branqueamento na textura final da batata frita. Somente quando o branqueamento foi associado à imersão das batatas em $\mathrm{NaCl}$ é que pôde-se observar aumento da crocância (BUNGER et al., 2003; PEDRESCHI et al., 2007).

Resultados semelhantes sobre a influência do revestimento foram obtidos por Suárez et al. (2008) e por Tavera Quiroz et al. (2012), visto que o revestimento de MC 1\% não modificou significativamente a textura de discos de massa e a crocância de batatas processadas na forma de chips, respectivamente. Por outro lado, Hua et al. (2015) observaram influência dos revestimentos compostos por diferentes concentrações de MC na força de compressão de batatas chips.

Com relação à cor da batata frita, por análise visual, foi observado que a batata BRS Ana, quando crua, é mais branca e menos amarela do que a Asterix. De acordo com Pereira et al. (2010), a época de cultivo influencia nas características da batata, podendo a mesma cultivar ser mais clara ou mais escura comparada a outra cultivar, dependendo da estação do ano e região de cultivo.

$\mathrm{Na}$ Tabela 2 são apresentados os valores médios de coloração das batatas fritas submetidas aos diferentes tratamentos. A batata BRS Ana controle apresentou luminosidade significativamente superior à batata Asterix controle. Porém, quando submetidas ao branqueamento (T1 e T4), houve redução da luminosidade em relação às amostras não branqueadas. A aplicação do revestimento (T2, T3 e T5, T6) aumentou a luminosidade da batata frita em relação ao tratamento em que as amostras foram submetidas somente ao branqueamento (T1 e T4), entretanto, não houve diferença significativa ( $p \leq 0,05)$ entre os resultados.

De acordo com os resultados dos valores de $a^{*}$ (Tabela 2), o branqueamento influenciou significativamente na diminuição da intensidade da coloração vermelha para as duas cultivares de batata. Foi possível observar que as batatas fritas controle (BRS Ana e ASTERIX) ficaram com aparência mais tostada do que as branqueadas em todas as condições testadas. Não houve diferença significativa nos resultados dos valores de $a^{*}$ para as amostras submetidas ao branqueamento e adicionadas de revestimento de $\mathrm{MC} 1$ e $2 \%$.

O branqueamento reduziu a intensidade dos valores de $b^{*}$. A redução foi significativa para os tratamentos em que a batata BRS Ana foi utilizada.
Ao comparar esses resultados com o teor de glicose das batatas in natura da cultivar BRS Ana $(0,096 \%)$ e da batata Asterix $(0,058 \%)$, percebe-se que não houve influência significativa $(p \leq 0,05)$ da concentração do açúcar redutor na coloração do produto frito, discordando assim de Márquez e Añón (1986), que concluíram que a cor da batata frita depende do conteúdo de açúcares redutores e aminoácidos ou proteínas que participam da reação de Maillard, além da temperatura e tempo de fritura.

Brown e Morales (1970) afirmaram que o processo de branqueamento favorece a cor da batata por lixiviar os açúcares solúveis que promovem o escurecimento. Entretanto, Troncoso et al. (2009) ao realizarem o branqueamento como pré-tratamento ao processo de fritura de fatias de batata, não observaram mudança nos parâmetros $L^{*} e a^{*}$. Já nos estudos realizados por Santis et al. (2007), pôde-se observar aumento da luminosidade da batata submetida ao branqueamento associado à imersão das batatas em solução de $\mathrm{NaCl}$. Diferente dos resultados obtidos neste trabalho, em que foi observada redução da luminosidade quando a batata foi submetida ao branqueamento associada à adição de sais.

Suárez et al. (2008) ao avaliarem a cor de discos de massa revestidos com MC 1\%, submetidos a diferentes tempos de fritura, não verificaram diferença significativa nos parâmetros avaliados entre as amostras revestidas e não revestidas. Já no estudo realizado por Hua et al. (2015), os parâmetros de cor avaliados em batata frita no formato chips foram influenciados pelos diferentes revestimentos compostos de MC 1\% e pectina de baixo teor de metoxilação, assim como a concentração de cálcio presente.

Os resultados da análise sensorial de preferência (Tabela 3) destacam que para os atributos sabor e textura não houve diferença significativa ( $p \leq 0,05)$ entre os tratamentos testados. Para o atributo cor, o tratamento da batata BRS Ana com branqueamento e com revestimento de $2 \%$ de $\mathrm{MC}$ foi o menos preferido, diferindo significativamente $(p \leq 0,05)$ dos demais.

Com relação à aparência para a batata BRS Ana, a preferência foi menor para os tratamentos utilizando a $\mathrm{MC}$, com diferença significativa apenas para a utilização de $2 \%$ de MC. Para a batata Asterix, a utilização de MC não influenciou na preferência entre os tratamentos.

O resultado da aceitação sensorial para o tratamento que obteve o melhor resultado de redução de absorção de gordura, tratamento T2, atingiu 85,9\% de aceitação. Conforme Dutcosky (2013), esse valor indica uma aceitação muito boa do produto. Tavera-Quiroz et al. (2012) observaram que os resultados da análise sensorial indicaram que os julgadores não conseguiram distinguir entre as batatas chips revestidas e não revestidas com 
Características físico-químicas e sensoriais de batata frita da cultivar BRS Ana branqueada e revestida com metilcelulose LUVIELMO, M. M. et al.

Tabela 3. Resultados do teste de ordenação de preferência para os atributos aparência, cor, sabor e textura.

\begin{tabular}{|c|c|c|c|c|c|c|c|c|c|}
\hline \multirow{2}{*}{$\begin{array}{c}\text { Cultivar } \\
\text { Batata }\end{array}$} & \multirow{2}{*}{ Tratamento } & \multicolumn{2}{|c|}{ Aparência } & \multicolumn{2}{|c|}{ Cor } & \multicolumn{2}{|c|}{ Sabor } & \multicolumn{2}{|c|}{ Textura } \\
\hline & & Ord. & Notas & Ord. & Notas & Ord. & Notas & Ord. & Notas \\
\hline \multirow{3}{*}{ BRS ANA } & BRANQ.(T1) & 4 & $196^{a}$ & 4 & $201^{a}$ & 3 & $178^{a}$ & 3 & $181^{a}$ \\
\hline & 1\%MC (T2) & 2 & $172^{\mathrm{ab}}$ & 2 & $180^{a}$ & 1 & $160^{a}$ & 5 & $196^{a}$ \\
\hline & 2\%MC (T3) & 1 & $123^{b}$ & 1 & $106^{b}$ & 2 & $173^{a}$ & 1 & $169^{a}$ \\
\hline \multirow{3}{*}{ ASTERIX } & BRANQ.(T4) & 3 & $187^{\mathrm{ab}}$ & 3 & $198^{a}$ & 4 & $183^{a}$ & 2 & $179^{a}$ \\
\hline & 1\%MC (T5) & 6 & $221^{a}$ & 6 & $217^{a}$ & 5 & $211^{a}$ & 6 & $197^{a}$ \\
\hline & 2\%MC (T6) & 5 & $214^{a}$ & 5 & $214^{a}$ & 6 & $217^{a}$ & 4 & $193^{a}$ \\
\hline
\end{tabular}

Resultado do método de Friedman e tabela de Newell e MacFarlane ao nível de significância de 5\%, escala (6 - mais preferida e 1 - menos preferida). BRS Ana: BRANQ (T1): batata com branqueamento e sem revestimento; $1 \% \mathrm{MC}$ (T2): batata com branqueamento e com revestimento de $1 \%$ de MC; $2 \%$ MC (T3): batata com branqueamento e com revestimento de $2 \%$ de MC; ASTERIX: BRANQ (T4): batata com branqueamento e sem revestimento; $1 \%$ MC (T5): batata com branqueamento e com revestimento de $1 \%$ de MC; $2 \%$ MC (T6): batata com branqueamento e com revestimento de $2 \%$ de MC.

MC. Já no estudo de Hua et al. (2015), de um modo geral, as batatas fritas sem revestimentos obtiveram os maiores escores para os atributos avaliados, assim como aceitação, em relação àquelas revestidas com MC ou pectina de baixo teor de metoxilação.

\section{Conclusão}

A batata BRS Ana, submetida ao branqueamento adicionado de $\mathrm{NaCl}$ e $\mathrm{CaCl}_{2}$ e adicionada de revestimento composto de $1 \%$ de metilcelulose, apresenta potencial de utilização na forma de batata chips por apresentar alto percentual de aceitação e menor percentual de absorção de gordura sem, contudo, influenciar na cor, força de quebra e preferência para os atributos aparência, sabor e textura.

\section{Agradecimentos}

Ao Dr. Arione da Silva Pereira pela doação das batatas utilizadas no experimento.

\section{Referências}

ALBERT, S.; MITTAL, G. S. Comparative evaluation of edible coatings to reduce fat uptake in a deep-fried cereal product. Food Research International, Toronto, v. 35, n. 5, p. 445-458, 2002.

BROWN, M. S.; MORALES, J. W. Determination of blanching conditions for frozen par-fried potatoes. American Potato Journal, Orono, v. 47, n. 9, p. 323-324, 1970.

BUNGER, A.; MOYANO, P.; RIOSECO, V. NaCl soaking treatment for improving the quality of french-fried potatoes. Food Research International, Oxford, v. 36, n. 2, p. 161-166, 2003. http://dx.doi. org/10.1016/S0963-9969(02)00131-X.

CUNNIFF, P. (Ed.). Official methods of analysis of the Association of Official Analytical Chemists. 16th ed. Washington: AOAC, 1995. 1094 p.

DEL RÉ, P. V.; JORGE, N. Comportamento de óleos vegetais em frituras descontínuas de produtos pré-fritos congelados.
Ciência e Tecnologia de Alimentos, Campinas, v. 26, n. 1, p. 56-63, 2006.

DIAS, P. D. B. Efeito da utilização de película e de diferentes formas de acondionamento na conservação pós-colheita e fritura de batatas minimamente processadas. 2011. $137 \mathrm{f}$. Dissertação (Mestrado em Ciência e Tecnologia de Alimentos)Escola Superior de Agricultura Luiz de Queiroz, Universidade de São Paulo, Piracicaba, 2011.

DUTCOSKY, S. D. Análise sensorial de alimentos. 4. ed. Curitiba: Editora Champagnat, 2013. 531 p.

FERNANDES, A. M.; SORATTO, R. P.; EVANGELISTA, R. M.; NARDIN, I. Qualidade físico-química e de fritura de tubérculos de cultivares de batata na safra de inverno. Horticultura Brasileira, Brasília, v. 28, n. 3, p. 299-304, 2010.

FREITAS, S. T.; BISOGNIN, D. A.; GÓMEZ, A. C. S.; SAUTTER, C. K.; COSTA, L. C.; RAMPELOTTO, M. V. Qualidade para processamento de clones de batata cultivados durante a primavera e outono no Rio Grande do Sul. Ciência Rural, Santa Maria, v. 36, n. 1, p. 80-85, 2006. http://dx.doi.org/10.1590/ S0103-84782006000100012.

GARAYO, J.; MOREIRA, R. Vacuum frying of potato chips. Journal of Food Engineering, Oakville, v. 55, n. 2, p. 181-191, 2002.

GARCíA, M. A.; FERRERO, C.; BÉRTOLA, N.; MARTINO, M.; ZARITZKI, N. Edible coatings from celullose derivatives to reduce oil uptake in fried foods. Innovative Food Science and Emerging Technologies, Berlin, v. 3, n. 4, p. 391-397, 2002.

HUA, X.; WAN, K.; YANG, R.; KANG, J.; YANG, H. Edible coatings from sunflower head pectin to reduce lipid uptake in fried potato chips. Food Science and Technology, Zurich, v. 62, n. 2, p. 1220-1225, 2015.

INSTITUTO BRASILEIRO DE GEOGRAFIA E ESTATÍSTICA IBGE. Levantamento sistemático da produção. Rio de Janeiro: IBGE, 2014.

JORGE, N.; LUNARDI, M. V. Influência dos tipos de óleo e tempos de fritura na perda de umidade e absorção de óleo em 
Características físico-químicas e sensoriais de batata frita da cultivar BRS Ana branqueada e revestida com metilcelulose LUVIELMO, M. M. et al.

batatas fritas. Ciências Agrotécnicas, Lavras, v. 29, n. 3, p. 635-641, 2005.

KIM, D. N.; LIM, J.; BAE, I. Y.; LEE, H. G. LEE. S. Effect of hydrocolloid coatings on the heat transfer and oil uptake during frying of potato strips. Journal of Food Engineering, Oakville, v. 102, n. 4, p. 317-320, 2011.

MÁRQUEZ, G.; AÑÓN, M. C. Influence of reducing sugars and amino acids in the color development of fried potatoes. Journal of Food Science, Chicago, v. 51, n. 1, p. 157-160, 1986. http:// dx.doi.org/10.1111/j.1365-2621.1986.tb10859.x.

MELLENA, M. Mechanism and reduction of fat uptake in deep-fat fried foods. Trends in Food Science \& Technology, Norwich, v. 14, n. 9, p. 364-373, 2003.

NELSON, N. A fotometric adaptation of Somogyi method for the determination of glucose. Journal of Biological Chemistry, Bethesda, v. 153, p. 375-380, 1944.

PEDRESCHI, F.; COCIO, C.; MOYANO, P.; TRONCOSO, E. Oil distribution in potato slices during frying. Journal of Food Engineering, Oakville, v. 87, n. 2, p. 200-212, 2008.

PEDRESCHI, F.; MOYANO, P. Oil uptake and texture development in fried potato slices. Journal of Food Engineering, Oakville, v. 70, n. 4, p. 557-563, 2005.

PEDRESCHI, F.; MOYANO, P.; SANTIS, N.; PEDRESCHI, R. Physical properties of pre-treated potato chips. Journal of Food Engineering, Oakville, v. 79, n. 4, p. 1474-1482, 2007.

PENA, A. L. Hidrocolóides: uso em alimentos. Food Ingredients, São Paulo, v. 1, p. 31-35, 1999.

PEREIRA, A. S. A evolução da batata no Brasil. Horticultura Brasileira, Brasília, v. 29, n. 2, p. 5701-5710, 2011.

PEREIRA, A. S.; BERTONCIN, O.; CASTRO, C. M.; DE MELO, P. E.; MEDEIROS, C. A. B.; HIRANO, E.; GOMES, C. B.; TREPTOW, R. O.; LOPES, C. A.; NAZARENO, N. X. R.; MACHADO, C. M. M.; BUSO, J. A.; OLIVEIRA, R. P.; UENO, B. BRS Ana: cultivar de batata de duplo propósito. Horticultura Brasileira, Brasília, v. 28, n. 4, p. 500-505, 2010.

PEREIRA, A. S.; BERTONCINI, O.; CASTRO, C. M.; HIRANO, E.; MELO, P. E.; MEDEIROS, C. A.; GOMES, C. B.; TREPTOW, R. O.; NAZARENO, N. X. R.; MACHADO, C. M. M.; LOPES, C. A.; KUHN, C. A.; BUSO, J. A.; OLIVEIRA, R. P.; UENO, B. BRS Ana: Cultivar de batata para fritas à francesa. Horticultura Brasileira, Brasília, v. 26, n. 2, p. S3829-S3833, 2008.
RIMAC-BRNCIC, S.; LELAS, V.; RADE, D.; SIMUNDI, B. Decreasing of oil absorption in potato strips during deep fat frying. Journal of Food Engineering, Oakville, v. 64, n. 2, p. 237-241, 2004.

SANTIS, N.; MENDOZA, F.; MOYANO, P.; PEDRESCHI, F.; DEJMEK, P. Soaking in a $\mathrm{NaCl}$ solution produce paler potato chips. Food Science and Technology, Zurich, v. 40, n. 2, p. 307-312, 2007.

STATISTIX 10. Data analysis software for researchers. Tallahassee: Analytical Software. Disponível em: <http://www. statistix.com/free-trial/>. Acesso em: 01 out. 2014.

SUÁREZ, R. B.; CAMPAÑONE, L. A.; GARCÍA, M. A.; ZARITZKY, N. E. Comparison of the deep frying process in coated and uncoated dough systems. Journal of Food Engineering, Oakville, v. 84, n. 3, p. 383-393, 2008.

TAVERA-QUIROZ, M. J.; URRIZA, M.; PINOTTI, A.; BERTOLA, $N$. Plasticized methylcellulose coating for reducing oil uptake in potato chips. Journal of the Science of Food and Agriculture, London, v. 92, n. 7, p. 1346-1353, 2012. http:// dx.doi.org/10.1002/jsfa.4704. PMid:22102087.

TRAN, M. T. T.; CHEN, X. D.; SOUTHERN, C. Reducing oil content of fried potato crisps considerably using a 'sweet' pre-treatment technique. Journal of Food Engineering, Oakville, v. 80, n. 2, p. 719-726, 2007.

TRONCOSO, E.; PEDRESCHI, F.; ZÚÑIGA, R. N. Comparative study of physical and sensory properties of pre-treated potato slices during vacuum and atmospheric frying. Food Science and Technology, Zurich, v. 42, n. 1, p. 187-195, 2009.

VENDRUSCOLO, J. L. S.; ZORZELLA, C. A. Processamento de batata (Solanum tuberosum L.): fritura. Pelotas: Emprapa Clima Temperado, 2002. $15 \mathrm{p}$.

ZENG, X.; CHENG, K. W.; DU, Y.; KONG, R.; LO, C.; CHU, I. K.; CHEN, F.; WANG, M. Activities of hydrocolloids as inhibitors of acrylamide formation in model. Food Chemistry, London, v. 121, n. 2, p. 424-428, 2010.

ZORZELLA, C. A.; VENDRUSCOLO, J. L. S.; TREPTOW, R. O.; ALMEIDA, T. L. Caracterização física, química e sensorial de genótipos de batata processados na forma de chips. Brazilian Journal of Food Technology, Campinas, v. 6, n. 1, p. 15-24, 2003. 